\title{
Coulomb effects in the spin-dependent contribution to the intrabeam scattering rate
}

\author{
V. M. Strakhovenko* \\ Budker Institute of Nuclear Physics, 630090, Novosibirsk, Russia
}

(Received 17 January 2010; published 31 January 2011)

\begin{abstract}
Coulomb effects in intrabeam scattering are taken into account in a way providing a correct description of the spin-dependent contribution to a beam loss rate. It allows one to calculate this rate for polarized $e^{ \pm}$beams at arbitrarily small values of $\delta \varepsilon / \varepsilon$, characterizing a relative change of the electron energy, $\varepsilon$, in the laboratory system during the scattering event.
\end{abstract}

DOI: 10.1103/PhysRevSTAB.14.012803

PACS numbers: 13.88.+e, 25.30.Bf, 12.20.Ds

\section{INTRODUCTION}

As is well known, elastic scattering of electrons in beams moving in a storage ring leads to a beam loss (Touschek effect). The longitudinal momentum $p_{\|}$of an electron is negligibly small in the beam rest system due to smallness of the beam energy spread. However, electron scattering may lead to energy changes in the laboratory system (LS), $\pm \delta \varepsilon$, exceeding the machine's energy acceptance. Then both electrons leave the beam and can be registered by some counters. The rate of such events depends on the beam polarization since the scattering cross section depends on the polarization of electrons. So, as was proposed in [1], the beam polarization can be measured by measuring that rate. A resonant depolarization technique for such measurements has been developed in Budker Institute of Nuclear Physics (BINP) since 1970 (see e.g. $[2,3])$. In this technique, a jump in the counting rate of Touschek electrons or positrons is measured. It occurs when an initially polarized beam is rapidly depolarized by applying a magnetic field with the frequency satisfying spin resonance conditions. As the resonant frequency depends in a certain way on the beam energy, the latter can be measured alongside with the beam polarization. Such a technique is routinely used in BINP for high accuracy absolute calibration of the beam energy (see e.g. [4,5]).

First spin effect calculations were carried out in [1] assuming that the betatron motion is one dimensional (flat beam) and that $\eta \ll 1$, where $\eta$ is a minimum value of $|\delta \varepsilon| / \varepsilon$ which enters the problem. Both limitations were formally overcome in [3]. In fact, results of [3] are correct only for $\eta \ll 1$ (see discussion in [6]). The scattering cross section used in both papers was obtained in the Born approximation. Accurate formulas for the scattering rate were derived in [6] within the same approximation. Coulomb effects were also considered in [6] but only for a flat beam. In the case of electron-electron interaction these effects are important at very small velocities $v$ in the center-of-mass system (c.m.s.) when $\alpha / v \gtrsim 1$ ( $\alpha$ is the fine-structure constant and $\hbar=c=1$ ). For such velocities

\footnotetext{
*v.m.strakhovenko@inp.nsk.su
}

the cross section is modified due to a corresponding change of the $e^{+}\left(e^{-}\right)$flux near the origin (see, e.g. [7]). In particular, the spin-dependent terms in the cross section are enhanced at $\alpha / v \gg 1$ for attraction and suppressed for repulsion (see discussion in [8]). In the case of intrabeam scattering we are just dealing with repulsion.

In the present paper, the scattering cross section of identical particles is modified in such a way that at $v \ll 1$ it becomes a nonrelativistic one (see, e.g. [7]) with Coulomb effects taken into account. The modified cross section passes into the expression obtained in the Born approximation at $\alpha / v \ll 1$, being thereby correct for any $v$. The condition $|\delta \varepsilon| / \varepsilon \geq \eta$ in LS is equivalent to the condition $\left|v_{\|}^{\prime}\right| \geq \eta$ in c.m.s. ( $v^{\prime}$ is an electron velocity after scattering). So, to get the rate of events with $|\delta \varepsilon| / \varepsilon \geq \eta$, the differential cross section, defined in c.m.s., should be integrated over the region where $\left|v_{\|}^{\prime}\right| \geq \eta$. The latter condition can be satisfied only if $v \geq \eta$. Thus $v \ll 1$ may enter the problem for $\eta \ll 1$. However, use of the modified differential cross section provides one with values of the integrated cross section, $\sigma_{\eta}$, which are correct for any $\eta$.

\section{RESULTS AND DISCUSSION}

Let us consider a collision of two electrons of the beam, having in LS momenta $\boldsymbol{p}_{1,2}$, energies $\boldsymbol{\varepsilon}_{1,2}$, and polarization vectors $\zeta_{1,2}$. It is convenient to calculate the scattering cross section in their c.m.s. A transition to that system is performed by the Lorentz transform with velocity $\boldsymbol{V}=\left(\boldsymbol{p}_{1}+\right.$ $\left.\boldsymbol{p}_{2}\right) /\left(\varepsilon_{1}+\varepsilon_{2}\right)$. Conditions $\varepsilon / m \gg 1$ and $\boldsymbol{p}_{\perp}^{2} / \varepsilon^{2} \ll 1$, where $m$ is the electron mass, are well fulfilled in storage rings. Then we have in c.m.s., where $\tilde{\boldsymbol{p}}_{1}+\tilde{\boldsymbol{p}}_{2}=0$,

$$
\tilde{p}_{1}^{\|} \simeq \tilde{\varepsilon} \frac{\varepsilon_{1}^{2}-\varepsilon_{2}^{2}}{4 \varepsilon_{1} \varepsilon_{2}} \sim \frac{1}{2} \tilde{\varepsilon}\left(\frac{\Delta \varepsilon_{b}}{\varepsilon_{b}}\right)
$$

where $\varepsilon_{b}$ is the mean beam energy. Perpendicular and parallel components of vectors are defined here with respect to the velocity $\boldsymbol{V}$, which direction practically coincides with the beam axis. In our problem, $\tilde{p}_{1}^{\|}$can be neglected due to 
smallness of the relative energy spread in the beam, $r=$ $\Delta \varepsilon_{b} / \varepsilon_{b} \ll 1$. More precisely, let $\tilde{p}_{1}^{\prime l}$ be the parallel component of the electron momentum in c.m.s. after scattering. Then the electron energy in LS becomes $\varepsilon_{1}^{\prime} \simeq$ $\varepsilon_{b}\left(1+\tilde{p}_{1}^{\prime \prime} / \tilde{\varepsilon}\right)$ and the relative energy change $d=$ $\delta \varepsilon_{1} / \varepsilon_{1} \simeq \tilde{p}_{1}^{\prime \prime} / \tilde{\varepsilon}$. As was explained above, we consider events where $|d| \geq \eta$ and scattered electrons leave the beam during one turn. The latter means that $\eta \gg r$. Otherwise, the beam lifetime would be too short. So, the minimum value of $\tilde{p}_{1}^{\prime \prime}$ which enters the problem is much larger than the initial value of $\tilde{p}_{1}^{\|}$. Self-consistently, the transverse momentum turns out to be much larger than the parallel one as $\tilde{p}_{1}^{\|} / \tilde{p}_{1 \perp} \sim r / v$ and $v \geq \eta$. In what follows we set $\tilde{p}_{1}^{\|}=0$, so that the electron energy in c.m.s. is $\tilde{\varepsilon}=\varepsilon_{q} \equiv \sqrt{\boldsymbol{q}^{2}+m^{2}}$, where $\boldsymbol{q}=\left(\boldsymbol{p}_{1 \perp}-\boldsymbol{p}_{2 \perp}\right) / 2=\tilde{\boldsymbol{p}}_{1 \perp}$.

We start from the well-known expression for the invariant event number in a collision of two beams with spatial densities $n_{1,2}(\boldsymbol{r})$ and momenta $\boldsymbol{p}_{1,2}$

$$
d n=I \frac{n_{1}(\boldsymbol{r}) n_{2}(\boldsymbol{r})}{\varepsilon_{1} \varepsilon_{2}} d \sigma d^{3} r d t, \quad I=\sqrt{\left(p_{1} p_{2}\right)^{2}-m^{4}} .
$$

In our case $\varepsilon_{1} \simeq \varepsilon_{2} \simeq \varepsilon_{b}$, the invariant $I \simeq 2 q \varepsilon_{q}, n_{1}(\boldsymbol{r})=$ $n_{2}(\boldsymbol{r}) \equiv n(\boldsymbol{r})$, and $d \sigma$ is the differential cross section for elastic ee scattering. To get the total rate of producing Touschek pairs, we have to integrate $d n$ in Eq. (1) over $\boldsymbol{r}$, calculate $\sigma_{\eta}$ by integrating $d \sigma$ over the region $\left|v_{\|}^{\prime}\right| \geq \eta$, and average over momentum and polarization distribution in the beam. The result should be divided by two as we are dealing with identical particles. For a Gaussian distribution over coordinates we have

$$
\int d^{3} r n^{2}(\boldsymbol{r})=\frac{N^{2}}{V_{b}}, \quad V_{b}=8 \pi^{3 / 2} \Delta_{x} \Delta_{z} \Delta_{\|},
$$

where $N$ is the total number of particles in the beam, $V_{b}$ is the beam volume, and $\Delta_{x}, \Delta_{z}$, and $\Delta_{\|}$are, respectively, the radial $(x)$, vertical $(z)$, and longitudinal ( II ) r.m.s. beam sizes. To average over momenta, the integral $\int d^{3} p_{1} d^{3} p_{2} f\left(\boldsymbol{p}_{1}\right) f\left(\boldsymbol{p}_{2}\right) q \varepsilon_{q} \sigma_{\eta}$ should be taken. Here $f(\boldsymbol{p})$ represents a momentum distribution in the beam. Since $\boldsymbol{p}_{1,2}$ enter $\sigma_{\eta}$ and the invariant $I$ only in the combination $\boldsymbol{q}=\left(\boldsymbol{p}_{1 \perp}-\boldsymbol{p}_{2 \perp}\right) / 2$, the integrals over $p_{1,2}^{\|}$and $\left(\boldsymbol{p}_{1 \perp}+\right.$ $\left.p_{2 \perp}\right)$ can be easily taken. After that the integral passes into $\int d^{2} q \sigma_{\eta}(\boldsymbol{q}) q \varepsilon_{q} F(\boldsymbol{q})$. Using a Gaussian type of $f(\boldsymbol{p})$ with r.m.s. parameters $\delta_{x}, \delta_{z}$, and $\delta_{\|}$, we obtain $F(\boldsymbol{q})=$ $\left(\pi \delta_{x} \delta_{z}\right)^{-1} \exp \left(-q_{x}^{2} / \delta_{x}^{2}-q_{z}^{2} / \delta_{z}^{2}\right)$ as the distribution over $q$. So the rate in LS, $\nu(\eta)$, reads

$$
\begin{aligned}
\nu(\eta) \equiv & \frac{d n}{d t}=\frac{N^{2}}{\pi \delta_{x} \delta_{z} V_{b} \varepsilon_{b}^{2}} \int d^{2} q \sigma_{\eta}(\boldsymbol{q}) q \varepsilon_{q} \\
& \times \exp \left(-q_{x}^{2} / \delta_{x}^{2}-q_{z}^{2} / \delta_{z}^{2}\right) .
\end{aligned}
$$

Now we pass to the differential cross section for elastic scattering of polarized electrons summed up over final spin states. Let us define in c.m.s. a coordinate system with basis vectors $\boldsymbol{e}_{3}=\boldsymbol{q} / q, \boldsymbol{e}_{2}=\boldsymbol{V} / V$, and $\boldsymbol{e}_{1}=\left[\boldsymbol{e}_{2} \times \boldsymbol{e}_{3}\right]$. The scattering angles $\vartheta$ and $\phi$ are defined in such a way that $\tilde{\boldsymbol{p}}^{\prime} / q=\boldsymbol{e}_{3} \cos \vartheta+\left(\boldsymbol{e}_{1} \cos \phi+\boldsymbol{e}_{2} \sin \phi\right) \sin \vartheta$.

In that notation the cross section reads

$$
\frac{d \sigma}{d \Omega^{\prime}}=\frac{\alpha^{2}}{2 \varepsilon_{q}^{2}}\left(F_{1}+F_{2}+F_{3}+F_{4}\right)
$$

where the functions $F_{i}$ are

$$
\begin{aligned}
& F_{1}=2 m^{4}\left[\frac{1}{t^{2}}+\frac{1}{u^{2}}+\frac{1+\boldsymbol{\zeta}_{1} \cdot \boldsymbol{\zeta}_{2}}{t u} \cos \left(\frac{\alpha}{2 v} \ln \frac{t}{u}\right)\right], \\
& F_{2}=8 q^{2} \varepsilon_{q}^{2}\left(\frac{1}{t^{2}}+\frac{1}{u^{2}}\right)+\frac{1}{2}\left(1+\boldsymbol{\zeta}_{1} \cdot \boldsymbol{\zeta}_{2}\right)\left(1-\frac{16 m^{2} q^{2}}{t u}\right)+\left(\frac{m^{2}}{q^{2}}-1\right) \boldsymbol{e}_{3} \cdot \boldsymbol{\zeta}_{1} \boldsymbol{e}_{3} \cdot \boldsymbol{\zeta}_{2}, \\
& F_{3}=\left[\frac{4 q^{2}\left(4 q^{2}+m^{2}\right)}{u t}-1\right]\left[\boldsymbol{e}_{2} \cdot \boldsymbol{\zeta}_{1} \boldsymbol{e}_{2} \cdot \boldsymbol{\zeta}_{2}-\frac{m}{q} \boldsymbol{e}_{1} \cdot\left[\boldsymbol{\zeta}_{1} \times \boldsymbol{\zeta}_{2}\right]\right], \\
& F_{4}=\left(\frac{4 q^{2} m^{2} v^{2}}{u t}-1\right)\left\{\sin ^{2} \phi\left[\frac{m^{2}}{q^{2}} \boldsymbol{e}_{2} \cdot \boldsymbol{\zeta}_{1} \boldsymbol{e}_{2} \cdot \boldsymbol{\zeta}_{2}-\boldsymbol{e}_{3} \cdot \boldsymbol{\zeta}_{1} \boldsymbol{e}_{3} \cdot \boldsymbol{\zeta}_{2}+\frac{m}{q} \boldsymbol{e}_{1} \cdot\left[\boldsymbol{\zeta}_{1} \times \boldsymbol{\zeta}_{2}\right]\right]+\frac{\cos ^{2} \phi}{v^{2}} \boldsymbol{e}_{1} \cdot \boldsymbol{\zeta}_{1} \boldsymbol{e}_{1} \cdot \boldsymbol{\zeta}_{2}\right\},
\end{aligned}
$$

where $t=-2 q^{2}(1-\cos \vartheta)$ and $u=-2 q^{2}(1+\cos \vartheta)$ are conventional kinematic variables. Functions in (5) are derived in the following way. We start from the result obtained in [9] in the Born approximation and unfold that in c.m.s. As was noted in [6], while unfolding in c.m.s. the invariants containing 4-vector of spin, one should remember that the unit spin vector $\zeta$ is not a Lorentz invariant. If in some reference frame an electron has the momentum $\boldsymbol{p}$, the energy $\varepsilon$, and the spin vector $\boldsymbol{\zeta}$, then in the reference frame moving with the velocity $\boldsymbol{V}$ the vector $\tilde{\zeta}$ reads

$$
\tilde{\zeta}=\zeta+\nu(\zeta \cdot[a \times b])-[\boldsymbol{\nu} \times \boldsymbol{b}](\zeta \cdot a)
$$




$$
\begin{aligned}
\boldsymbol{\nu} & =\boldsymbol{V} / V, & \boldsymbol{a} & =\boldsymbol{p} \frac{\gamma_{V}-1}{\varepsilon+m}-\boldsymbol{V} \gamma_{V}, \\
\boldsymbol{b} & =\frac{[\boldsymbol{p} \times \boldsymbol{\nu}]}{\boldsymbol{\varepsilon}^{\prime}+m}, & \gamma_{V} & =\frac{1}{\sqrt{1-V^{2}}} .
\end{aligned}
$$

As a next step, the terms giving a nonrelativistic limit (for $\boldsymbol{v}=\boldsymbol{q} / \varepsilon_{q} \ll 1$ ) are separated up. In Eq. (4) such terms are combined in the function $F_{1}$ which is proportional to $v^{-4}$. The rest functions in Eq. (4) contain terms having at least an excess factor of $v^{2}$ as compared with $F_{1}$. They represent in this limit the relativistic corrections. In the Born approximation, we have for $F_{1}^{B}$

$$
F_{1}^{B}=2 m^{4}\left[\frac{1}{t^{2}}+\frac{1}{u^{2}}+\frac{1+\boldsymbol{\zeta}_{1} \cdot \boldsymbol{\zeta}_{2}}{t u}\right] .
$$

The last term in Eq. (8) $(\propto 1 / t u)$ addresses exchange interaction (see, e.g. [7]). It originates from interference of two Coulomb amplitudes addressing scattering at $(\vartheta, \phi)$ and $(\pi-\vartheta, \pi+\phi)$. The phase of the Coulomb amplitude is proportional to $\alpha$ and is ignored in the Born approximation. Taking that phase and thereby the Coulomb corrections into account results in the appearance of the factor $\cos \left(\frac{\alpha}{2 v} \ln \frac{t}{u}\right)$ by the exchange term in Eq. (8). In that way we pass from $F_{1}^{B}$ in Eq. (8) to $F_{1}$ in Eq. (5). That modification provides the correct nonrelativistic limit of the cross section (4). Strictly speaking, the remaining functions in Eq. (4) are also changed by the Coulomb corrections. However, since the latter become apparent only in the nonrelativistic limit, where the terms given by functions $F_{2,3,4}$ are small as compared with corresponding terms in $F_{1}$, in our problem we can use expressions for $F_{2,3,4}$ obtained in the Born approximation.

Let us emphasize that in certain problems Coulomb effects might be important also in the terms representing relativistic corrections. For example, to estimate the beam depolarization during the intrabeam scattering, one should calculate a probability of spin flip transitions. The spin dependence of the nonrelativistic spin flip cross section is completely due to the exchange interaction. In full agreement with the sense of that interaction, it allows only the simultaneous spin flip of both electrons, at which the initial spin directions should be opposite. Such transitions do not change the beam polarization. Therefore, relativistic corrections, addressing direct spin-spin and spin-orbit coupling, should be considered. As is shown in [8], Coulomb effects may be very important in that case.

Passing to the calculation of $\sigma_{\eta}$, we integrate the differential cross section (4) over the region $\left|v_{\|}^{\prime}\right| \geq \eta$. In terms of the angles $\vartheta$ and $\phi$, the latter condition reads $\sin ^{2} \phi \sin ^{2}$ $\vartheta \geq \lambda^{2}$, where $\lambda=\eta / v \leq 1$. The only change in $\sigma_{\eta}$ as compared to $\sigma_{\eta}^{B}$ comes from the exchange term in $F_{1}$. Namely, the integral $\int d \Omega^{\prime}(t u)^{-1} \Theta\left(\sin ^{2} \phi \sin ^{2} \vartheta-\lambda^{2}\right)=$ $\frac{\pi}{q^{4}} \ln \frac{1}{\lambda}$ passes into $\int d \Omega^{\prime}(t u)^{-1} \cos \left(\frac{\alpha}{2 v} \ln \frac{t}{u}\right) \Theta\left(\sin ^{2} \phi \sin ^{2} \vartheta-\right.$ $\left.\lambda^{2}\right) \equiv \frac{\pi}{q^{4}} f(\lambda, \xi)$. Here $\Theta(x)$ is the step function and

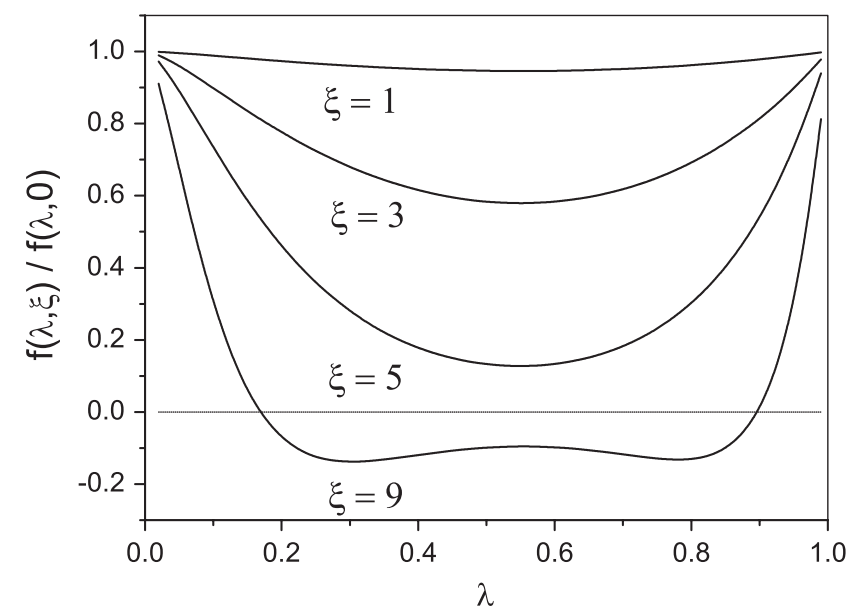

FIG. 1. Ratio of the function $f(\lambda, \xi)$ to its limit value $f(\lambda, 0)=\ln 1 / \lambda$.

$$
\begin{aligned}
f(\lambda, \xi) & =\frac{2}{\pi} \int_{0}^{\operatorname{Arcosh}(1 / \lambda)} d x \cos (\lambda \xi x) \arccos (\lambda \cosh x) \\
& \equiv \frac{2}{\pi \lambda \xi} \int_{0}^{\arccos \lambda} d x \sin \left[\lambda \xi \operatorname{Arcosh}\left(\frac{\cos x}{\lambda}\right)\right]
\end{aligned}
$$

where $\xi=\alpha / \eta$ and $\operatorname{Arcosh} z=\ln \left(z+\sqrt{z^{2}-1}\right)$. Shown in Fig. 1 is the ratio of the function $f(\lambda, \xi)$ to its limit in the Born approximation, $f(\lambda, 0)=\ln 1 / \lambda$. As is seen on Fig. 1, at $\xi=1$ the function $f(\lambda, \xi)$ practically coincides with its limit at $\xi \rightarrow 0$. Thus Coulomb effects are negligible in our problem for $\eta \geq \alpha$. At $\xi \gg 1$ the function $f(\lambda, \xi)$ is rapidly oscillating and the corresponding integral over $\lambda(q)$ in (3) falls off as $\xi^{-2}$.

Let the beam polarization be directed along a unit vector $\boldsymbol{s}$. We choose that direction as the quantization axis while performing averaging over electron polarizations. Then we obtain for the combination $\zeta_{1}^{i} \zeta_{2}^{j}$ which enter the cross section: $\left\langle\zeta_{1}^{i} \zeta_{2}^{j}\right\rangle=P^{2} s^{i} s^{j}$, where $P$ is the beam polarization degree. By definition, $P=\left(N_{\uparrow}-N_{\downarrow}\right) / N$, where $N_{\uparrow}$ and $N_{\downarrow}$ are numbers of electrons with spin projections onto the quantization axis $\pm 1 / 2$, respectively. The final form of $\sigma_{\eta}$ reads

$$
\sigma_{\eta}(\boldsymbol{q})=\frac{\pi \alpha^{2}}{q^{4} \varepsilon_{q}^{2}} G(\boldsymbol{q}) \Theta(1-\lambda),
$$

where

$$
\begin{aligned}
G(\boldsymbol{q})= & A_{0}+q^{2} A_{1}+P^{2}\left[q^{2} s_{\|}^{2} A_{\|}+q^{2}\left(s_{x}^{2}+s_{z}^{2}\right) A_{2}\right. \\
& \left.+\left(q_{x}^{2}-q_{z}^{2}\right)\left(s_{z}^{2}-s_{x}^{2}\right) A_{3}\right] .
\end{aligned}
$$

Here 


$$
\begin{aligned}
A_{0}= & \left(2 q^{2}+m^{2}\right)^{2}\left(\frac{1}{\lambda^{2}}-1\right)-m^{4}\left(1+P^{2}\right) f(\lambda, \xi), \\
A_{1}= & q^{2}(1-\lambda)-4 m^{2} \ln \frac{1}{\lambda}, \\
A_{\|}= & \left(1+v^{2}\right)\left[\left(4 \varepsilon_{q}^{2}-m^{2}\right) \ln \frac{1}{\lambda}-\frac{m^{2}}{2}\left(1-\lambda^{2}\right)\right] \\
& -2 q^{2}(1-\lambda)+A_{1},, \\
A_{2}= & m^{2}\left(\frac{m^{2}}{2 \varepsilon_{q}^{2}}-4\right) \ln \frac{1}{\lambda}+\frac{\left(2 q^{2}+m^{2}\right)^{2}}{4 \varepsilon_{q}^{2}}\left(1-\lambda^{2}\right), \\
A_{3}= & \frac{m^{2}}{2}\left(1+v^{2}\right)\left[\ln \frac{1}{\lambda}+\frac{1}{2}\left(1-\lambda^{2}\right)\right]-2 m^{2}(1-\lambda) .
\end{aligned}
$$

Remember that $\lambda=\eta / v, v=q / \varepsilon_{q}$, and $f(\lambda, \xi)$ is defined by Eq. (9). Substituting the obtained expression for $\sigma_{\eta}$ into Eq. (3), we have for the rate

$$
\begin{aligned}
\nu(\eta)= & \frac{\alpha^{2} N^{2}}{\varepsilon_{b}^{2} V_{b} \delta_{x} \delta_{z}} \int \frac{d^{2} q}{q^{3} \varepsilon_{q}} G(\boldsymbol{q}) \exp \left(-q_{x}^{2} / \delta_{x}^{2}\right. \\
& \left.-q_{z}^{2} / \delta_{z}^{2}\right) \Theta(1-\lambda) .
\end{aligned}
$$

If we substitute $\ln 1 / \lambda$ for $f(\lambda, \xi)$ in $A_{0}$, Eq. (12) passes into Eq. (5) of [6].

Expression (12) represents the rate of producing in the beam a pair of electrons with relative energy change $\pm \delta \varepsilon / \varepsilon_{b}$ which module is larger than $\eta$. It has a local character as the beam parameters and the polarization direction $s$ may vary along the orbit. An interval of $\eta$, which is important in measurements of the rate, is determined by the size and position of counters. Corresponding values of $\eta$ may be small or not. For any interrelation between $\delta_{x}, \delta_{z}$, and $\eta m$, the numerical integration in Eq. (12) is very simple. It is straightforward for flat or round beams and for any $\eta$.

If Eq. (12) is applied to estimate the beam lifetime, we deal with small $\eta \ll 1$. In that case all terms in $G(\boldsymbol{q})$ except $A_{0}$ can be neglected. Going from the integration over $q$ to the integration over $\lambda$, we obtain for $\eta \ll 1$

$$
\begin{aligned}
\nu(\eta)= & \frac{2 \pi r_{e}^{2} N^{2} m^{2}}{\gamma_{b}^{2} V_{b} \delta_{x} \delta_{z} \eta^{2}} \int_{\eta}^{1} d z\left\{\left(\frac{1+z^{2}}{1-z^{2}}\right)^{2} g\left(\frac{z^{2}}{1-z^{2}}\right)\right. \\
& \left.-\eta\left[1+\left(1+P^{2}\right) f(z, \xi)\right] g\left(\frac{\eta^{2}}{z^{2}-\eta^{2}}\right)\right\}
\end{aligned}
$$

where $\gamma_{b}=\varepsilon_{b} / m$ and

$$
g(Y)=\exp \left(-Y \beta_{+}^{2}\right) I_{0}\left(Y \beta_{-}^{2}\right), \quad \beta_{ \pm}^{2}=\frac{m^{2}}{2}\left(\frac{1}{\delta_{z}^{2}} \pm \frac{1}{\delta_{x}^{2}}\right)
$$

Here $I_{0}(x)$ is the modified Bessel function. Note that the rate (13) is independent of the beam polarization direction, $s$.

Let Touschek electrons with relative energy change larger than $\eta$ be registered by some counter. A jump in the counting rate is observed when initially polarized beam

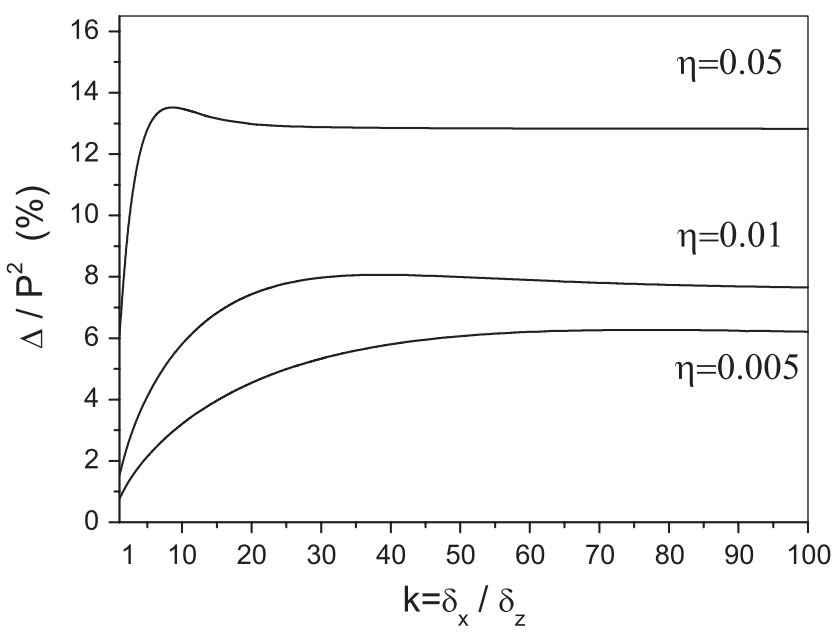

FIG. 2. Dependence of maximal jump $\Delta / P^{2}$ on $k=\delta_{x} / \delta_{z}$ at $\delta_{x}=0.5 m \simeq 0.26 \mathrm{MeV} / c$.

is depolarized. The jump $\Delta$ is usually defined as $\Delta=1-$ $\nu\left(\eta, P^{2}\right) / \nu(\eta, 0)$. In Fig. 2 the maximal possible jump $\Delta / P^{2}$ is shown as a function of $k=\delta_{x} / \delta_{z}$. It was calculated using Eq. (13) for several small values of $\eta$ at $\delta_{x}=$ $0.5 m \equiv 0.26 \mathrm{MeV} / c$. From Fig. 2, the jump increases with $\eta$ and the regime of the flat beam, where $\Delta / P^{2}$ becomes independent of $k$ is achieved earlier for larger $\eta$. From Eq. (12) we conclude that in our problem such a regime is realized at arbitrary $\eta$ for $\delta_{z} \ll q_{0}$ and $\delta_{x} \gtrsim q_{0}$, where $q_{0}=\eta m / \sqrt{1-\eta^{2}}$ is the lower limit of integration over $q$ in Eq. (12). If both $\delta_{z}$ and $\delta_{x}$ are much smaller than $q_{0}$, the rate (12) is strongly suppressed being proportional to $\exp \left(-q_{0}^{2} / \delta_{\max }^{2}\right)$, where $\delta_{\max }=\max \left(\delta_{x}, \delta_{z}\right)$. For the flat beam and small $\eta$ we obtain from Eq. (13)

$$
\nu(\eta)=\frac{2 \sqrt{\pi} r_{e}^{2} N^{2} m}{\gamma_{b}^{2} V_{b} \delta_{x} \eta^{2}}\left\{\ln \frac{2}{\eta}-\frac{3}{2}-\frac{c(\xi)}{4}\left(1+P^{2}\right)+B\left(\frac{m}{\delta_{x}}\right)\right\},
$$

where

$$
\begin{aligned}
B(z)= & \sqrt{\pi}\left\{\left(\frac{2}{z}+z\right)[1-\Phi(z)] \exp \left(z^{2}\right)\right. \\
& \left.-\int_{0}^{z} d x[1-\Phi(x)] \exp \left(x^{2}\right)\right\} \\
\Phi(z)= & \frac{2}{\sqrt{\pi}} \int_{0}^{z} d x \exp \left(-x^{2}\right), \\
c(\xi)= & \frac{4}{\xi} \int_{0}^{1} d x J_{1}\left(x \xi \operatorname{Arcosh} \frac{1}{x}\right)
\end{aligned}
$$

here $J_{1}(u)$ is the Bessel function. At $\xi \rightarrow 0$ the function $c(\xi) \rightarrow 1$ and it is suppressed at $\xi \gg 1$, where $c(\xi) \simeq$ $4 /\left(\xi^{2} \ln 2 \xi\right)$. Such power suppression at $\xi \gg 1$ is an ordinary Coulomb effect for repulsion. Note that there are some misprints in Eqs. (7) and (8) of [6] representing the same quantities as Eqs. (15) and (16) above. 
To conclude, the cross section of intrabeam scattering is modified to take into account Coulomb effects. It allows one to calculate the beam loss rate for polarized $e^{ \pm}$beams at arbitrary values of $\eta$. It turns out that Coulomb effects in the rate are negligible for $\eta \geq \alpha$. To our knowledge, all earlier measurements of the rate were performed just for $\eta>\alpha$ and therefore they can be correctly estimated using Eq. (5) of [6].

\section{ACKNOWLEDGMENTS}

This work was supported in part by Grant No. 09-02-00024 of the Russian Foundation for Basic Research.
[1] V. N. Baier and V. A. Khose, At. Energ. 25, 440 (1968).

[2] V. N. Baier, Usp. Fiz. Nauk 105, 3 (1971).

[3] S. I. Serednyakov, A.N. Skrinsky, G. M. Tumaykin, and Yu. M. Shatunov, Zh. Eksp. Teor. Fiz. 71, 2025 (1976).

[4] S. I. Serednyakov et al., Phys. Lett. B 66, 102 (1977).

[5] V.E. Blinov et al., Nucl. Instrum. Methods Phys. Res., Sect. A 494, 81 (2002).

[6] V. N. Baier, V. M. Katkov, and V. M. Strakhovenko, Dokl. Acad. Nauk SSSR 241, 797 (1978).

[7] L.D. Landau and E.M. Lifshits, Quantum Mechanics, Nonrelativistic Theory (Pergamon, Oxford, 1965).

[8] A. I. Milstein, S. G. Salnikov, and V. M. Strakhovenko, Nucl. Instrum. Methods Phys. Res., Sect. B 266, 3453 (2008).

[9] G. W. Ford and C. J. Mullin, Phys. Rev. 108, 477 (1957); 110, 1485 (1958) 